\title{
Does microbleed predict haemorrhagic transformation after acute atherothrombotic or cardioembolic stroke?
}

S-H Lee, B-S Kang, N Kim, et al.

$J$ Neurol Neurosurg Psychiatry 2008 79: 913-916 originally published online January 10,2008

doi: 10.1136/jnnp.2007.133876

Updated information and services can be found at:

http://jnnp.bmj.com/content/79/8/913.full.html

\begin{tabular}{|c|c|}
\hline \multirow[b]{2}{*}{ References } & These include: \\
\hline & $\begin{array}{l}\text { This article cites } 24 \text { articles, } 20 \text { of which can be accessed free at: } \\
\text { http://jnnp.bmj.com/content/79/8/913.full.html\#ref-list-1 }\end{array}$ \\
\hline \multirow[b]{2}{*}{$\begin{array}{l}\text { Email alerting } \\
\text { service }\end{array}$} & $\begin{array}{l}\text { Article cited in: } \\
\text { http://jnnp.bmj.com/content/79/8/913.full.html\#related-urls }\end{array}$ \\
\hline & $\begin{array}{l}\text { Receive free email alerts when new articles cite this article. Sign up in the } \\
\text { box at the top right corner of the online article. }\end{array}$ \\
\hline \multirow[t]{2}{*}{ Topic collections } & Articles on similar topics can be found in the following collections \\
\hline & $\begin{array}{l}\text { Neuroimaging ( } 355 \text { articles) } \\
\text { Stroke (9696 articles) }\end{array}$ \\
\hline
\end{tabular}

Notes

To order reprints of this article go to:

http://jnnp.bmj.com/cgi/reprintform

To subscribe to Journal of Neurology, Neurosurgery \& Psychiatry go to:

http://jnnp.bmj.com/subscriptions 


\title{
Does microbleed predict haemorrhagic transformation after acute atherothrombotic or cardioembolic stroke?
}

\author{
S-H Lee, ${ }^{1,2}$ B-S Kang, ${ }^{1}$ N Kim, ${ }^{1}$ J-K Roh ${ }^{1}$
}

${ }^{1}$ Department of Neurology, Seoul National University Hospital, Seoul, Republic of Korea; ${ }^{2}$ Clinical Research Center for Stroke, Clinical Research Institute, Seoul National University Hospital, Seoul, Republic of Korea

Correspondence to: Jae-Kyu Roh, Department of Neurology, Seoul National University Hospital, 28 Yongondong, Jongno-gu, Seoul 110-

744, Republic of Korea; rohjk@ snu.ac.kr

Received 1 September 2007 Revised 30 November 2007 Accepted 5 December 2007 Published Online First 10 January 2008

\begin{abstract}
Objectives: Cerebral microbleeds (MBs) are known to be indicative of bleeding-prone microangiopathy and may predict incident intracerebral haemorrhage. However, there is controversy concerning the causal relationship between the presence of MBs and haemorrhagic transformation (HTf) after ischaemic stroke.
\end{abstract}

Methods: Of the 1034 patients with acute ischaemic stroke who were consecutively admitted to our hospital, 377 patients with stroke due to large-artery atherothrombosis or cardioembolism were selected for participation in this study. We examined the MBs using T2*weighted gradient-echo MRI performed within 24 hours after admission, and the incidence of HTf was assessed using follow-up brain MRI during the hospitalisation period.

Results: Of the 377 patients with stroke, 234 were male $(62.1 \%)$ and the mean age was $66.2 \pm 11.7$ years. MBs were initially found in 109 patients (28.9\%), and newly incident HTf was noted during the hospitalisation period in 74 patients (19.6\%). The presence of MBs was not increased in the patients with HTf (24.3\% vs. $30.0 \%$ in the patients without HTf; $p=0.331$ ). In addition, the number of MBs was not higher in the patients with HTf $(0.7 \pm 1.5$ vs. $1.8 \pm 8.1 ; p=0.234)$. This lack of significance between MBs and HTf persisted after stratification by stroke mechanism.

Conclusions: This study suggests that underlying MBs do not predict incident HTf after acute ischaemic stroke. The clinical significance of MBs should be differentially evaluated according to the type of disease (intracerebral haemorrhage vs. HTf).

Microbleeds (MBs) visualised on brain $\mathrm{T}^{*}$ weighted gradient-echo (GRE) MRI are indicative of chronic cerebral microangiopathy caused by various conditions, including hypertension and ageing. ${ }^{1-3}$ It has been suggested that the lesions may predict subsequent haemorrhagic events such as intracerebral haemorrhage ${ }^{4-6}$ and haemorrhagic transformation (HTf) of ischaemic stroke. ${ }^{78} \mathrm{HTf}$ was determined to be one of the most important risk factors leading to poor outcome in patients with acute ischaemic stroke. However, the MBs do not appear to predict an incident HTf after thrombolysis in acute ischaemic stroke. ${ }^{9}$ Irrespective of thrombolysis, a prior study showed that the presence of MBs may be associated with HTf after acute ischaemic stroke, ${ }^{8}$ but the results have not yet been confirmed. In this study, we sought to investigate whether MBs that are present at baseline are associated with subsequent HTf in patients with acute ischaemic stroke by analysing data on a larger, consecutive series of patients with specific types of ischaemic stroke associated with haemorrhagic transformation-large-artery atherothombosis and cardioembolism.

\section{METHODS}

A total of 1034 acute ischaemic stroke patients who had been admitted to Seoul National University Hospital within 7 days after ictus were consecutively enrolled in this study between October 2002 and March 2006. Among the patients, subjects with the following conditions were excluded from this study according to the classification of Trial of Org 10172 in Acute Stroke Treatment (TOAST): ${ }^{11}$ patients with only transient ischaemic attack $(n=110)$, stroke due to smallvessel disease $(n=289)$, stroke of undetermined aetiology $(n=184)$, or stroke of other determined aetiology $(n=15)$. Patients whose work-ups were not complete due to various reasons were further excluded. Therefore, our study population consisted of a total of 377 patients with stroke due to large-artery atherothombosis $(\mathrm{n}=217)$ and cardioembolism $(n=160)$.

Baseline demographic and clinical characteristics were collected at admission and included age at onset, gender, hypertension (previous use of antihypertensive medication, systolic blood pressure $>140 \mathrm{mmHg}$ or diastolic blood pressure $>90 \mathrm{mmHg}$ at discharge), diabetes (previous use of antidiabetic medication, fasting blood glucose $>7.0 \mathrm{mmol} / \mathrm{L}$ or postprandial blood glucose after 2 hours $>11.1 \mathrm{mmol} / \mathrm{L}$ at discharge), hyperlipidaemia (previous use of lipid-lowering agents, total cholesterol $>6.0 \mathrm{mmol} / \mathrm{L}$ or low-density lipoprotein [LDL] cholesterol $>4.14 \mathrm{mmol} / \mathrm{L}$ at admission), history of transient ischaemic attack or stroke, previous use of antiplatelet or anticoagulant medications, systolic and diastolic blood pressure level at admission, glucose level, total cholesterol level, prolonged prothrombin time or activated partial thromboplastin time, initial National Institutes of Health Stroke Scale (NIHSS) score, and type of treatment during the acute stage (thrombolysis, anticoagulant or antiplatelet medications).

All participants underwent brain MRI before the initiation of thrombolytic or anti-thrombotic therapy (within 24 hours after admission) and follow-up brain GRE MRI. The MRI studies were performed using a 1.5-Tesla superconducting magnet (GE Medical System, Milwaukee, WI, USA) The standardised MRI protocol consisted of axial T2-weighted spin echo (repetition time (TR)/echo 
time (TE), 2500-4500/80-112 ms; flip angle, 20 ; slice thickness, $5 \mathrm{~mm}$; gap width, $2 \mathrm{~mm}$ ), axial GRE sequences (TR/TE, 200$500 / 15 \mathrm{~ms}$; flip angle, $20^{\circ}$; slice thickness, $5 \mathrm{~mm}$; gap width, $2 \mathrm{~mm}$ ) and diffusion-weighted imaging (repetition time (TR)/ echo time (TE), 4000/73 ms; flip angle, $90^{\circ}$; slice thickness, $5 \mathrm{~mm}$; gap width, $2 \mathrm{~mm}$ ). We examined the $\mathrm{MBs}$ present at baseline by analysing the GRE image obtained at admission. $\mathrm{MBs}$ were defined as focal homogeneous hypointense areas with a diameter of 2-5 $\mathrm{mm}$. Signal loss lesions secondary to globus pallidus calcification or thrombus in the cerebral artery were excluded. We determined the number and location of $\mathrm{MBs}$ in each patient. The cerebral lesions ( $\mathrm{MBs}$ and ischaemic stroke lesions) were located in the cortical areas, deep grey matter, brain stem and cerebellum. Old ischaemic or haemorrhagic stroke lesions were also evaluated. Leukoaraiosis was classified as being absent, or present as a punctate, early confluent or confluent abnormality (as seen on T2-weighted MR images) according to the method proposed by Fazekas et al., ${ }^{12}$ and early confluent or confluent lesions were compared in this study. HTf was identified by follow-up brain GRE MRI and, according to the prespecified criteria, ${ }^{13}$ the severity of HTf was graded into four categories: haemorrhagic infarction (HI)-1 (small petechiae along the margins of the infarct); HI-2 (more confluent petechiae within the infarct but without space-occupying effect); parenchymal hematoma (PH)-1 (blood clots not exceeding $30 \%$ of the infarct area); and $\mathrm{PH}-2$ (blood clots exceeding $30 \%$ of the infarct area with significant spaceoccupying effect).

Spearman's rank correlation analysis was used to test interobserver reliabilities of $\mathrm{MB}$ numbers. Associations between variables and incident HTf were examined by Pearson's $\chi^{2}$ test, Fisher's exact test and Student's t test. The numbers of MBs were compared according to the presence or the severity of HTf using a nonparametric analysis (Mann-Whitney $U$ test), because the numbers of $\mathrm{MBs}$ were not normally distributed (Kolmogorov-Smirnov test; $p<0.05$ ). All analyses were performed using SPSS statistical software (Version 12.0.1). In all tests, $\mathrm{p}<0.05$ was noted as significant.

\section{RESULTS}

The inter-observer reliability of $\mathrm{MB}$ numbers between the reading of observer 1 (S-HL) and observer 2 (B-SK) was high $(\mathrm{r}=0.87, \mathrm{p}<0.001)$. Of the 377 patients included in this study, 234 patients were male $(62.1 \%)$ and the mean age of the subjects was $66.2 \pm 11.7$ years. There were 232 patients with hypertension (61.5\%), 118 with diabetes (31.3\%) and 64 with hyperlipidaemia (17.0\%). Prior to admission, 100 patients $(26.5 \%)$ had a history of transient ischaemic attack or stroke, 40 patients $(10.6 \%)$ had been prescribed antiplatelet agents and 27 patients $(7.2 \%)$ had been prescribed anticoagulants. Incident HTf was found in 74 patients (19.6\%), and symptomatic HTf was found in 13 patients (3.4\%). Patients with incident HTf were likely to receive thrombolytic treatment $(p<0.001)$ and to have a higher NIHSS score $(p<0.001)$ (table 1$)$. The interval between the first imaging and the follow-up imaging ranged from 1 to 17 days (mean, $6.0 \pm 7.3$ days; median, 8 days).

Baseline MBs revealed by GRE MRI were present in 109 patients $(28.9 \%)$ and were most frequently located in the cortical area (70 patients, $18.6 \%$ ). The number of lesions ranged from 0 to 122. As illustrated in table 2, among the patients with incident HTf during the hospitalisation period, baseline MBs were found in 18 patients $(24.3 \%)$ and the association between $\mathrm{MBs}$ and HTf was not significant $(\mathrm{p}=0.331)$. Reclassification of the subjects according to the location of the MBs (cortical, deep grey matter, brain stem and cerebellum) did not affect the results. When we limited the outcome to symptomatic HTf, an association between the presence of MBs and symptomatic HTf was not found ( $p=0.637$ by Pearson's $\chi^{2}$ test).

We also found that there was no association between the number of MBs and the incident HTf ( $p=0.234$ by MannWhitney $U$ test) (table 2). According to the severity of HTf, no significant differences were found (No HTf, 1.8 \pm 8.1 ; HI-1, 1.1 $\pm 2.2 ; \mathrm{HI}-2,0.4 \pm 1.0 ; \mathrm{PH}-1,0.4 \pm 1.0 ; \mathrm{PH}-2,0.6 \pm 1.2 ; \mathrm{p}>0.05$ in all comparisons using Mann-Whitney $U$ test). Among the radiological variables, patients with stroke lesions located in the grey matter were more likely to have incident HTf during hospitalisation $(\mathrm{p}=0.001)$.

We performed two subgroup analyses based on stroke mechanism and stroke location in order to identify any significant relationships among the subgroups. In the initial analysis based on stroke mechanism (large-artery atherothombosis and cardioembolism), neither subgroup showed significant association between MBs and incident HTf (data not shown).There were also no significant results in the subgroup analysis based on stroke location (cortical and deep grey matter) (data not shown).

\section{DISCUSSION}

In this study, underlying MBs observed on the initial GRE MR images were not associated with incident HTf occurring during hospitalisation after acute ischaemic stroke. The presence, number and location of $\mathrm{MBs}$ were not related to the incidence of HTf, and there were no significant differences among the groups classified according to HTf severity. In addition, no significant relationships were found between HTf and any of the subgroups. Stroke location (cortical-subcortical or deep grey matter), use of thrombolytic agents and high initial NIHSS score were associated with the subsequent haemorrhagic transformations.

$\mathrm{MBs}$ indicate previous extravasation of blood, signifying bleeding-prone cerebral microangiopathy..$^{5-714}$ It has been suggested that $\mathrm{MBs}$ may predict the incidence of intracerebral haemorrhage. ${ }^{41516} \mathrm{~A}$ previous study of 41 patients who underwent intra-arterial thrombolysis demonstrated that the presence of baseline MBs could be a risk factor for symptomatic HTf. ${ }^{7}$ On the other hand, a subsequent retrospective study of 44 patients who had received intravenous thrombolysis did not replicate the positive association between $\mathrm{MBs}$ and HTf reported in the previous study. ${ }^{10}$ Furthermore, a recent prospective study, which was a part of the DEFUSE trial, showed that MBs were detected in 11 out of 70 patients with intravenous thrombolysis, and none of the 11 patients with $\mathrm{MBs}$ at baseline had symptomatic HTf compared with 7 of the 59 patients who did not have baseline MBs (11.9\%). ${ }^{9}$ Considering these results, MBs are not likely to be associated with risk of HTf after thrombolysis. In this context, the predictive value of $\mathrm{MBs}$ for subsequent HTf should be reevaluated in all types of HTf. A study on the effects of MBs on early cerebral bleeding after acute ischaemic stroke reported that MBs were found in 20 of 100 patients with acute ischaemic stroke and in 10 of 26 patients with HTf $(p<0.001) .{ }^{8}$ However, the study had an important limitation: in 11 of the 20 patients with MBs, GRE MRI was performed after the initiation of either systemic thrombolysis or anti-thrombotic treatment (mainly high-dose heparin), both of which may contribute to MBs or HTf formation. ${ }^{8}{ }^{17}$ In this study, all of the GRE MRIs were performed before the initiation of thrombolytic or antithrombotic therapy, and they did not confirm the positive 
Table 1 Demographic, clinical and laboratory findings in patients with stroke due to large-artery atherothombosis or cardioembolism

\begin{tabular}{llll}
\hline & HTf & \\
\cline { 2 - 2 } & Absent $(\mathbf{n}=\mathbf{3 0 3})$ & Present (n= 74) & p Value \\
\hline Demographic & & & \\
$\quad$ Gender (male) & $188(62.0 \%)$ & $46(62.2 \%)$ & 0.985 \\
$\quad$ Age at admission (years) & $66.1 \pm 12.2$ & $66.8 \pm 9.8$ & $0.643^{*}$ \\
Clinical & & & \\
Hypertension & $186(61.4 \%)$ & $46(62.2 \%)$ & 0.902 \\
Diabetes & $92(30.4 \%)$ & $26(35.1 \%)$ & 0.427 \\
Hyperlipidaemia & $54(17.8 \%)$ & $10(13.5 \%)$ & 0.376 \\
Smoking & $100(33.0 \%)$ & $25(33.8 \%)$ & 0.898 \\
History of transient ischaemic attack & $21(6.9 \%)$ & $1(1.4 \%)$ & $0.093^{\dagger}$ \\
History of stroke & $62(20.5 \%)$ & $16(21.6 \%)$ & 0.897 \\
Previous use of antiplatelet agents & $32(10.6 \%)$ & $8(10.8 \%)$ & 0.950 \\
Previous use of anticoagulation & $19(6.3 \%)$ & $8(10.8 \%)$ & 0.174 \\
Initial NIHSS score & $5.5 \pm 5.9$ & $10.3 \pm 7.1$ & $<0.001^{*}$ \\
Acute treatment & & & \\
$\quad$ Thrombolysis & $19(6.3 \%)$ & $20(27.0 \%)$ & $<0.001$ \\
$\quad$ Antiplatelet agents & $115(38.0 \%)$ & $31(41.9 \%)$ & 0.533 \\
$\quad$ Anticoagulation & $179(59.1 \%)$ & $29(39.2 \%)$ & 0.002 \\
Laboratory & & & \\
Glucose (mmol/L) & $6.44 \pm 2.19$ & $6.75 \pm 2.10$ & $0.294^{*}$ \\
Total cholesterol (mmol/L) & $4.72 \pm 1.05$ & $4.55 \pm 0.99$ & $0.208^{*}$ \\
Systolic blood pressure (mmHg) & $151.0 \pm 25.5$ & $149.9 \pm 25.0$ & $0.745^{*}$ \\
Diastolic blood pressure (mmHg) & $86.7 \pm 15.1$ & $85.8 \pm 13.2$ & $0.636^{*}$ \\
Mean blood pressure (mmHg) & $108.1 \pm 17.1$ & $107.2 \pm 15.1$ & $0.657^{*}$ \\
Prolonged PT/aPTT (\%) & $63(20.8 \%)$ & $16(21.6 \%)$ & 0.875 \\
\hline Peas & & & \\
\hline
\end{tabular}

Pearson's $\chi^{2}$ test, ${ }^{*}$ Student $\mathrm{t}$ tests and 'Fisher's exact test were used.

aPTT, activated partial thromboplastin time; HTf, haemorrhagic transformation; NIHSS, National Institutes of Health Stroke Scale; PT, prothrombin time.

association between the presence of baseline MBs and subsequent incidence of HTf.

The underlying histopathologies of intracerebral haemorrhage include rupture of penetrating arterioles damaged by hyaline degeneration and microaneurysm formation caused by longstanding hypertension or ageing. ${ }^{18-20}$ However, the pathophysiological mechanism of HTf formation after acute ischaemic stroke is different from that of intracerebral haemorrhage.

Table 2 Analysis of radiological variables

\begin{tabular}{|c|c|c|c|}
\hline & \multicolumn{2}{|l|}{ HTf } & \multirow[b]{2}{*}{ p Value } \\
\hline & Absent $(n=303)$ & Present $(n=74)$ & \\
\hline \multicolumn{4}{|l|}{ Presence of MBs } \\
\hline Whole brain & $91(30.0 \%)$ & $18(24.3 \%)$ & 0.331 \\
\hline Cortical & $59(19.5 \%)$ & $11(14.9 \%)$ & 0.361 \\
\hline Deep grey matter & $55(18.2 \%)$ & $10(13.5 \%)$ & 0.344 \\
\hline Brain stem & $35(11.6 \%)$ & $6(8.1 \%)$ & 0.394 \\
\hline Cerebellum & $24(7.9 \%)$ & $3(4.1 \%)$ & 0.247 \\
\hline Number of MBs & $1.8 \pm 8.1$ & $0.7 \pm 1.5$ & $0.234^{*}$ \\
\hline \multicolumn{4}{|l|}{ Location of stroke } \\
\hline Cortical & $208(68.6 \%)$ & $59(79.7 \%)$ & 0.060 \\
\hline Deep grey matter & $71(23.4 \%)$ & $32(43.2 \%)$ & 0.001 \\
\hline Brain stem & $45(14.9 \%)$ & $6(8.1 \%)$ & 0.128 \\
\hline Cerebellum & $52(17.2 \%)$ & $17(23.0 \%)$ & 0.246 \\
\hline Leukoaraiosis (early confluent or confluent) & $79(26.1 \%)$ & $19(25.7 \%)$ & 0.944 \\
\hline Absent & $115(38.0 \%)$ & $38(51.4 \%)$ & \\
\hline Punctate & $109(36.0 \%)$ & $17(23.0 \%)$ & \\
\hline Early confluent & $48(15.8 \%)$ & $15(20.3 \%)$ & \\
\hline Confluent & $31(10.2 \%)$ & $4(5.4 \%)$ & \\
\hline \multicolumn{4}{|l|}{ Old stroke lesion } \\
\hline All stroke & $96(31.7 \%)$ & $21(28.4 \%)$ & 0.582 \\
\hline Ischaemic stroke & $90(29.7 \%)$ & $19(25.7 \%)$ & 0.493 \\
\hline Haemorrhagic stroke & $10(3.3 \%)$ & $2(2.7 \%)$ & 0.793 \\
\hline
\end{tabular}

Pearson's $\chi^{2}$ test and *Mann-Whitney $\mathrm{U}$ test were used.

HTf, haemorrhagic transformation; MBs, microbleeds. 
Ischaemic injury to the microvasculature in extensive brain infarction is central to the risk of parenchymal haemorrhage following thrombolytic treatment in stroke. ${ }^{21}$ An experimental study demonstrated a correlation between the development of HTf and the loss of basal lamina architecture. ${ }^{22}$ Because the histopathological findings of $\mathrm{MBs}$ are similar to those of symptomatic intracerebral haemorrhage, $\mathrm{MBs}$ may not be associated with development of HTf in acute ischaemic stroke, as found in this study.

There were some caveats of this study. First, our study was conducted in a retrospective manner, and brain imaging was not performed using a predetermined protocol. Despite this limitation, we were able to determine the presence of incident HTf because follow-up brain imaging was performed in most of the patients (1001 out of 1034; 97\%). Second, this study did not evaluate the long-term effects of HTf, which is a major poor prognostic factor in acute stroke. Third, this study did not include patients with stroke due to small-vessel occlusion because a rarity of incident HTf in this subtype of stroke might have influenced the study results. Fourth, to focus on the association between the MBs and HTf, we did not conduct a multivariate analysis for identification of independent factors. Finally, the development of HTf was more frequent in cardioembolic stroke than in strokes due to other mechanisms. ${ }^{23}{ }^{24}$ Our result showed a similar tendency (cardioembolism, $33 \%$, 53/161; large-artery atherothombosis, 10\%, 22/219); however, there was no association between the presence of $\mathrm{MBs}$ and incident HTf after stratification by stroke mechanism.

This study suggests that underlying MBs may not predict incident HTf in acute ischaemic stroke. MBs reflect bleedingprone microangiopathy in the brain, but do not predict all forms of subsequent bleeding. The predictive values of CMBs should be differentially evaluated according to the type of disease (intracerebral haemorrhage vs. HTf).

Funding: This study was supported by grants from the Korea Health 21 RqD Project, Ministry of Health and Welfare, Republic of Korea (A060263).

Competing interests: None.

Ethics approval: Ethics approval was obtained.

\section{REFERENCES}

1. Lee SH, Bae HJ, Yoon BW, et al. Low concentration of serum total cholesterol is associated with multifocal signal loss lesions on gradient-echo magnetic resonance imaging: analysis of risk factors for multifocal signal loss lesions. Stroke 2002;33:2845-9
2. Lee SH, Park JM, Kwon SJ, et al. Left ventricular hypertrophy is associated with cerebral microbleeds in hypertensive patients. Neurology 2004;63:16-21.

3. Roob G, Schmidt R, Kapeller P, et al. MRI evidence of past cerebral microbleeds in a healthy elderly population. Neurology 1999:52:991-4.

4. Fan YH, Zhang L, Lam WW, et al. Cerebral microbleeds as a risk factor for subsequent intracerebral hemorrhages among patients with acute ischemic stroke. Stroke 2003;34:2459-62.

5. Fazekas F, Kleinert R, Roob G, et al. Histopathologic analysis of foci of signal loss on gradient-echo $\mathrm{T}^{*}$-weighted MR images in patients with spontaneous intracerebral hemorrhage: evidence of microangiopathy-related microbleeds. AJNR Am J Neuroradiol 1999;20:637-42.

6. Lee SH, Kim BJ, Roh JK. Silent microbleeds are associated with volume of primary intracerebral hemorrhage. Neurology 2006;66:430-2.

7. Kidwell CS, Saver JL, Villablanca JP, et al. Magnetic resonance imaging detection of microbleeds before thrombolysis: an emerging application. Stroke 2002;33:95-8.

8. Nighoghossian $\mathbf{N}$, Hermier $\mathbf{M}$, Adeleine $\mathrm{P}$, et al. Old microbleeds are a potential risk factor for cerebral bleeding after ischemic stroke: a gradient-echo $2^{*}$-weighted brain MRI study. Stroke 2002;33:735-42.

9. Kakuda W, Thijs VN, Lansberg MG, et al. Clinical importance of microbleeds in patients receiving IV thrombolysis. Neurology 2005;65:1175-8.

10. Derex L, Nighoghossian N, Hermier M, et al. Thrombolysis for ischemic stroke in patients with old microbleeds on pretreatment MRI. Cerebrovasc Dis 2004;17:23841.

11. Adams HP Jr, Bendixen BH, Kappelle LJ, et al. Classification of subtype of acute ischemic stroke. Definitions for use in a multicenter clinical trial. TOAST. Trial of Org 10172 in Acute Stroke Treatment. Stroke 1993;24:35-41.

12. Fazekas F, Chawluk JB, Alavi A, et al. MR signal abnormalities at 1.5 T in Alzheimer's dementia and normal aging. AJR Am J Roentgenol 1987;149:351-6.

13. Hacke W, Kaste M, Fieschi $C$, et al. Intravenous thrombolysis with recombinant tissue plasminogen activator for acute hemispheric stroke. The European Cooperative Acute Stroke Study (ECASS). JAMA 1995;274:1017-25.

14. Jeerakathil T, Wolf PA, Beiser A, et al. Cerebral microbleeds: prevalence and associations with cardiovascular risk factors in the Framingham Study. Stroke 2004;35:1831-5

15. Lee SH, Bae HJ, Kwon SJ, et al. Cerebral microbleeds are regionally associated with intracerebral hemorrhage. Neurology 2004;62:72-6.

16. Greenberg SM, Eng JA, Ning M, et al. Hemorrhage burden predicts recurrent intracerebral hemorrhage after lobar hemorrhage. Stroke 2004;35:1415-20.

17. Koennecke HC. Cerebral microbleeds on MRI: prevalence, associations, and potential clinical implications. Neurology 2006;66:165-71.

18. Fisher CM. Pathological observations in hypertensive cerebral hemorrhage. J Neuropathol Exp Neurol 1971;30:536-50.

19. Fisher CM. Cerebral miliary aneurysms in hypertension. Am J Pathol 1972;66:31330.

20. Cole FM, Yates PO. Comparative incidence of cerebrovascular lesions in normotensive and hypertensive patients. Neurology 1968;18:255-9.

21. del Zoppo GJ, von Kummer R, Hamann GF. Ischaemic damage of brain microvessels: inherent risks for thrombolytic treatment in stroke. J Neurol Neurosurg Psychiatry 1998;65:1-9.

22. Hamann GF, Okada Y, del Zoppo GJ. Hemorrhagic transformation and microvascular integrity during focal cerebral ischemia/reperfusion. J Cereb Blood Flow Metab 1996;16:1373-8.

23. Kidwell CS, Saver JL, Carneado J, et al. Predictors of hemorrhagic transformation in patients receiving intra-arterial thrombolysis. Stroke 2002;33:717-24.

24. Molina CA, Montaner J, Abilleira S, et al. Timing of spontaneous recanalization and risk of hemorrhagic transformation in acute cardioembolic stroke. Stroke 2001;32:1079-84. 